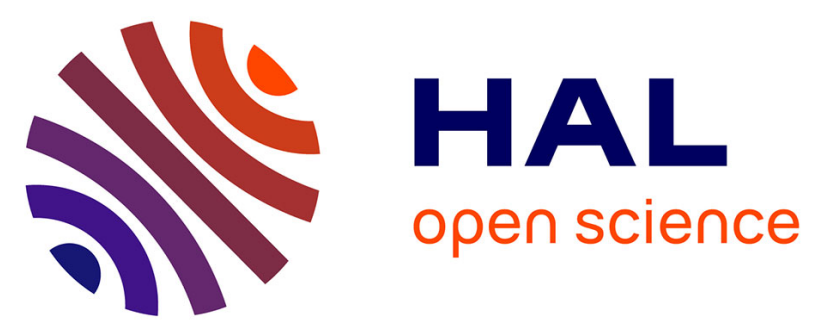

\title{
Dessertes TGV et tourismes urbain et d'affaires : entre attentes dans les villes de France et renforcement de la double centralité parisienne
}

\author{
Marie Delaplace, Julie Perrin
}

\section{To cite this version:}

Marie Delaplace, Julie Perrin. Dessertes TGV et tourismes urbain et d'affaires : entre attentes dans les villes de France et renforcement de la double centralité parisienne. RTS Recherche Transports Sécurité, 2013, Grande vitesse ferroviaire et territoires, 2013 (03), pp.177-191. 10.4074/S0761898013003038a . hal-01670630

\section{HAL Id: hal-01670630 \\ https://hal.science/hal-01670630}

Submitted on 21 Dec 2017

HAL is a multi-disciplinary open access archive for the deposit and dissemination of scientific research documents, whether they are published or not. The documents may come from teaching and research institutions in France or abroad, or from public or private research centers.
L'archive ouverte pluridisciplinaire HAL, est destinée au dépôt et à la diffusion de documents scientifiques de niveau recherche, publiés ou non, émanant des établissements d'enseignement et de recherche français ou étrangers, des laboratoires publics ou privés. 


\title{
Dessertes TGV et tourismes urbain et d'affaires : entre attentes dans les villes de France et renforcement de la double centralité parisienne
}

\author{
Linking high-speed rail services with urban and business tourism: \\ french cities' expectations vs. reinforcement of the dual centrality of Paris
}

\author{
Marie Delaplace · Julie Perrin \\ Reçu le 13 octobre 2012 ; accepté le 20 septembre 2013 \\ (C) IFSTTAR et Éditions NecPlus 2013
}

\begin{abstract}
Résumé En France, l'extension des dessertes à grande vitesse ferroviaire s'accompagne d'attentes significatives dans les villes desservies, en termes de développement des tourismes urbain et d'affaires. Étant caractérisés par des courts séjours, ces derniers peuvent tirer profit d'une desserte TGV dès lors que les équipements nécessaires et des aménités sont présents. Or ces attentes reposent sur un présupposé implicite mais néanmoins systématique : les dessertes TGV devraient permettre de contribuer à la « mise en tourisme » des villes françaises, en captant des touristes franciliens. En termes de tourisme, les effets de polarisation souvent associés aux dessertes TGV n'existeraient pas. Pourtant, l'autre bout de la ligne, ce cœur du réseau qu'est Paris-Ile-de-France et qui s'est renforcée au fur et à mesure de l'extension des dessertes, est aussi une destination
\end{abstract}

Cet article a bénéficié de recherches menées dans le cadre, d'une part, d'un contrat PREDIT financé par l'ADEME sur les enjeux et opportunités des dessertes ferroviaires à grande vitesse en termes de développement économique local et de développement durable et, d'autre part, d'une bourse CIFRE portée par le Comité Départemental $\mathrm{du}$ Tourisme de Seine-et-Marne sur la liaison entre dessertes TGV et tourisme d'affaires en Île-de-France. Les propos n'engagent néanmoins que leurs auteurs. Les auteurs remercient également les referees pour la qualité de leurs remarques.

\author{
Marie Delaplace $(\triangle)$ \\ Université de Paris-Est Marne-la-Vallée (UPEM) \\ IFU- EA 3482 Lab'Urba \\ Cité Descartes, 5, Bd Descartes \\ 77420 Champs sur marne \\ e-mail : marie.delaplace@univ-mlv.fr \\ Julie Perrin $(\bowtie)$ \\ Université de Paris-Est Marne-la-Vallée \\ (UPEM)/ Seine-et-Marne Tourisme \\ École des Ponts - UMR LVMT \\ 6-8 Avenue Blaise Pascal, Champs sur Marne \\ 77455 Marne-la-Vallée Cedex 2 \\ e-mail : julie.perrin@enpc.fr
}

touristique majeure. La centralité touristique de Paris ne peut-elle être également renforcée?

Mots clés TGV · tourismes urbain et d'affaires · villes françaises $\cdot$ Paris-Ile-de-France $\cdot$ flux touristiques

Abstract In France, the expansion of High-Speed Rail (HSR) makes cities expecting positive effects in terms of urban and business tourism development. Given that these forms of tourism are characterised by shorter stays, they could benefit from HSR services, whenever necessary facilities and amenities are present. But these expectations are based on the implicit and systematic assumption that HSR service could favour the tourism development in regional French cities by drawing tourists from the greater Paris. In terms of tourism development, the polarisation effects of HSR service would be largely absent. However Paris is also in the heart of the French High-Speed Line network and its place has been strengthened with the development of High-Speed Lines and services since the 1980s. Moreover, Paris is a major tourism destination. Could the Paris' tourist centrality be reinforced too?

Keywords High Speed Rail (HSR) · urban and business tourisms · French regional cities · Paris metropolitan area . tourists' flows

\section{Classification JEL : R $11 \cdot$ R $12 \cdot$ R $42 \cdot$ R $58 \cdot$ L 83}

Dans les villes françaises nouvellement desservies, de nombreuses attentes sont formulées concernant le développement du tourisme suite à l'arrivée d'une desserte ferroviaire à grande vitesse. Dans certains cas, c'est un développement du tourisme en général qui est évoqué, sans que soit précisé de quel tourisme il s'agit $[1,2]$. Toutefois, ce sont globalement les tourismes urbain et d'affaires qui 
suscitent le plus d'intérêt [3,4,5,6,7]. Si ces attentes ont évolué en 30 ans de desserte TGV en France ${ }^{1}$, un élément semble cependant généralement oublié : les dessertes TGV irriguent certes des villes françaises, mais elles participent également, en France, au renforcement du cœur du réseau, l'Ile-de-France et la capitale Paris. Ainsi ces attentes reposent sur un présupposé implicite mais néanmoins systématique : les dessertes TGV devraient permettre de capter des touristes franciliens. Mais si les dessertes peuvent être une source potentielle de développement des tourismes urbain et d'affaires dans les villes desservies, ne peuvent-elles également dynamiser ces tourismes dans la région dont la desserte est renforcée, c'est-à-dire Paris et l'Ile-de-France?

L'objectif de cet article est ainsi de questionner la liaison entre desserte TGV et développement des tourismes urbain et d'affaires du point de vue des attentes exprimées. Cet article met en évidence que dans les villes françaises, ces dernières reposent sur une vision univoque des flux touristiques envisagés. Alors que les dessertes TGV sont parfois accusées de polariser le développement, ces craintes ne sont pas évoquées dans le champ du tourisme. De surcroît, paradoxalement, ces attentes sont quasiment absentes à Paris et l'éventuel bénéfice de ces dessertes pour la destination Paris-Ile-de-France n'est pas questionné.

À partir d'une analyse de la littérature grise, nous mettons ainsi en évidence que Paris est analysée de façon quasiexclusive comme une émettrice de clientèle touristique. Cette absence du point de vue des villes françaises trouve un écho tout aussi surprenant en Ile-de-France : la question des dessertes TGV et de leur intérêt pour le développement touristique de Paris a longtemps été limitée. Pour autant, c'est bien la destination Paris qui a été progressivement renforcée et c'est également Paris et plus généralement aujourd'hui la destination Paris-Ile-de-France qui domine en matière de tourisme urbain et d'affaires.

\section{Dynamisme des tourismes urbain et d'affaires et dessertes TGV : entre attentes dans les villes françaises et évidence en Ile-de-France}

En 2012, 197 gares françaises et 56 gares européennes sont desservies par la grande vitesse ferroviaire française ${ }^{2}$ (Fig. 1). Avec le développement du réseau et l'extension des dessertes, des attentes spécifiques ont émergé dans les

\footnotetext{
${ }^{1} \mathrm{TGV}$, qui est une marque, ne concerne que la grande vitesse ferroviaire commercialisée par la SNCF, ce qui constitue l'essentiel de notre propos dans cet article. La distinction avec une desserte européenne (Eurostar, Thalys etc.) sera précisée, le cas échéant.

${ }^{2}$ Soit par une desserte TGV soit par une desserte co-opérée par la SNCF via ses partenariats avec d'autres opérateurs européens.
}

villes nouvellement desservies : celles-ci espèrent capter de nouveaux flux, en particulier touristiques.

Dans les villes desservies, c'est une clientèle de tourisme urbain et de tourisme d'affaires ${ }^{3}$ qui est visée, alors qu'à Paris aucune attente spécifique n'a vraiment émergé. Ces attentes importantes dans les villes françaises laisseraient à penser que les effets de polarisation parfois craints au sujet de la grande vitesse ferroviaire ne se produiraient pas en matière de tourisme.

\section{Des tourismes urbain et d'affaires qui se sont développés}

Les attentes liées au développement touristique se sont concentrées sur les tourismes urbain et d'affaires. Bien que distinctes, ces deux formes de tourisme sont généralement envisagées conjointement dans le cadre de stratégie globale. En effet, si les tourismes urbain de loisirs et d'affaires sont de nature différente, ils s'interpénètrent néanmoins dans la ville [9] et particulièrement dans les capitales [10] et ce pour plusieurs raisons.

Premièrement, se répartissant sur la fin de semaine pour le premier et sur les jours ouvrés pour le second, ils sont de fait complémentaires en termes de développement d'une destination.

Deuxièmement, les frontières entre touriste de loisirs et touriste d'affaires sont dans une certaine mesure poreuses. Les infrastructures utilisées ne sont qu'en partie spécifiques ; certains équipements touristiques comme les infrastructures de transport sont communs. Les politiques d'attractivité d'une destination visent bien souvent la complémentarité entre les deux types de publics puisque celle-ci permet de rentabiliser certains investissements.

Troisièmement, des processus d'induction du tourisme d'affaires vers des pratiques touristiques classiques peuvent être relevés : touriste d'affaires dans la semaine, le professionnel se mue en touriste d'agrément le week-end [11,12].

Quatrièmement, le tourisme urbain comme le tourisme d'affaires sont des tourismes de court séjour ( $c f$. infra), ce qui, dans les deux cas, confère à la qualité et la rapidité des transports vers la destination un rôle important.

Ainsi, et si ce sont des formes de tourisme différentes, la complémentarité entre pratiques professionnelles et pratiques touristiques nous conduit à les analyser en parallèle quant au rôle que peuvent jouer les dessertes TGV dans leur développement.

\footnotetext{
${ }^{3}$ Une desserte TGV peut également permettre une valorisation des tourismes de destination (stations du littoral, de montagne, etc.) mais plus difficilement pour le tourisme vert, en raison de la rupture de charge engendrée et de la nécessité d'un moyen de locomotion sur place [8].
} 


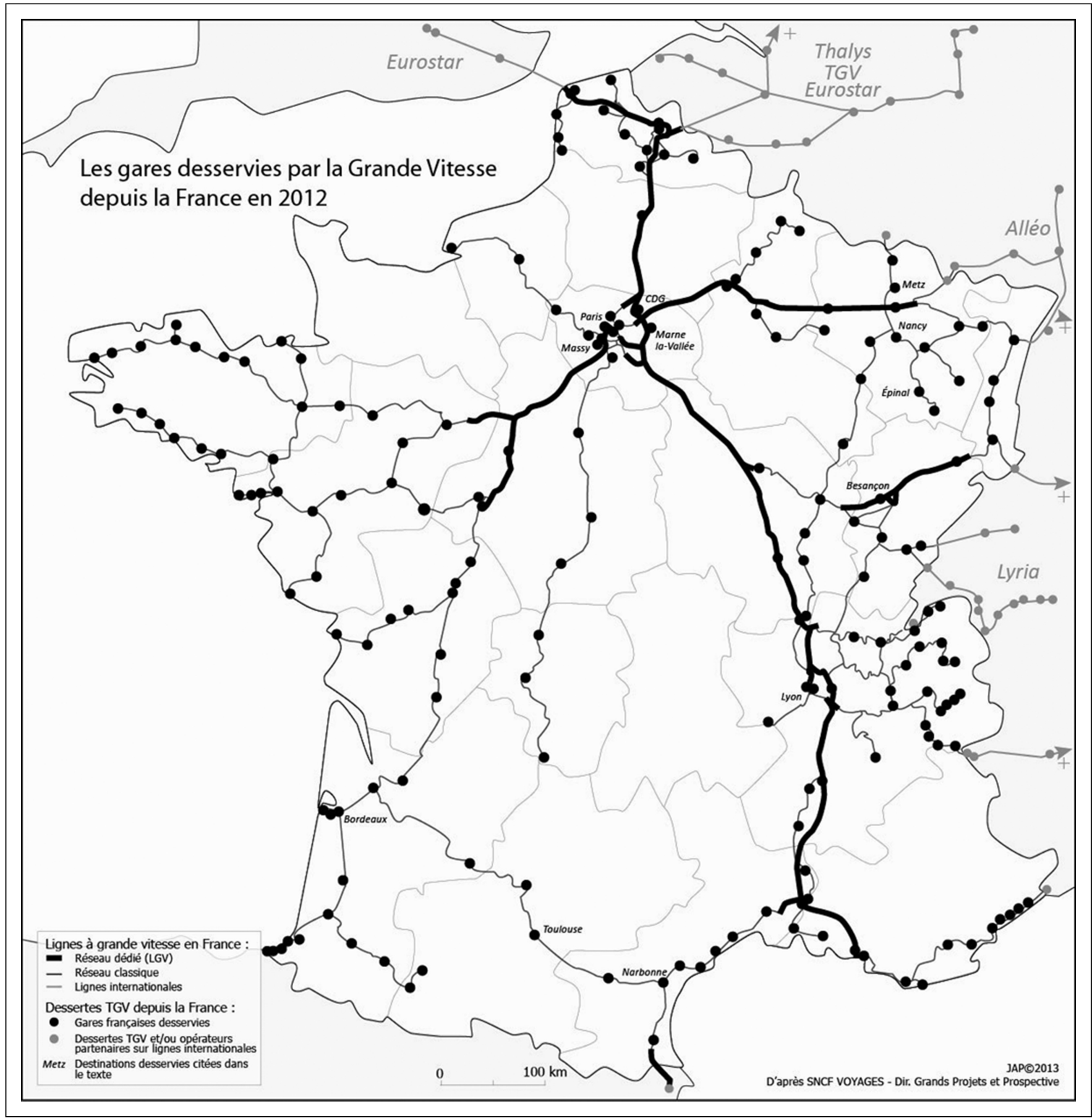

Fig. 1 Les gares françaises et européennes desservies par la grande vitesse ferroviaire française en $2012^{4}$

Si le tourisme urbain est une des formes de tourisme les plus anciennes et les plus pratiquées, il est sans doute également une des plus difficiles à appréhender $[13,14]$. Les premières Assises nationales du tourisme urbain tenues en 1988 le définissaient comme «l'ensemble des ressources et activités touristiques implantées en ville, stations mises à part, et proposées aux visiteurs extérieurs [...] à des fins de loisirs, d'affaires et autres motifs » [15]. Les activités qu'il implique sont par conséquent diverses (visites de musées, de monuments, gastronomie, shopping, événementiel, etc.). Si ces activités n'ont pas nécessairement de rapport entre elles, leur association permet de former une destination spécifique que le touriste vient découvrir.
En France, le tourisme urbain s'est développé de façon importante depuis les années 1970 avec la réduction $\mathrm{du}$ temps de travail et le fractionnement des temps de vacances. En 2007, il représentait 53 millions de voyages et 218,5 millions de nuitées ${ }^{5}$ pour la clientèle française. Le poids des chambres situées dans un espace urbain augmente également, passant de $68,5 \%$ en 1999 à 70,6 \% en 2007 [17]. C'est le plus souvent un tourisme de court séjour et de

4 Cette carte reprend les dessertes annoncées par la SNCF. L'objet n'est pas ici de qualifier le niveau de desserte (international, national, intersecteur, saisonnier, périodique) mais de représenter les grands axes de diffusion de la desserte en France et dans les territoires limitrophes. ${ }^{5}$ Depuis cette date et jusqu'en 2010, il a légèrement diminué [16]. 
fin de semaine ( 2 à 3 jours) réalisé par des personnes seules ou des couples appartenant à des professions et catégories socioprofessionnelles diversifiées ayant, pour certaines, une sensibilité aux prix assez importante.

La définition du tourisme d'affaires qui regroupe des activités diversifiées, fait également débat ${ }^{6}$. Selon la définition d'Atout France, l'organisme en charge de l'observation et de la promotion du tourisme en France, et animateur du Club Français du tourisme d'affaires réceptif (CFTAR), le tourisme d'affaires comprend à la fois les congrès, conventions, conférences, colloques ou cymposium, céminaires ou workshop, ou encore les visites de salons, de foires, permettant de nouer des contacts commerciaux, de promouvoir des produits ou services ou de réaliser des ventes, ou enfin les incentives ${ }^{7}$. Ce type de tourisme est caractérisé par des déplacements individuels et/ou collectifs de court séjour en semaine, effectué par des professions et catégories socioprofessionnelles à forts revenus avec une sensibilité moins marquée aux prix ${ }^{8}$, d'autant que ces déplacements ne sont pas financés par ceux qui se déplacent mais par leurs organisations. Il est également caractérisé par une tendance au raccourcissement de la durée moyenne des séjours [18].

Le tourisme d'affaires s'est également développé en France, avec une évolution récente toutefois plus erratique dans la mesure où il est extrêmement sensible à la conjoncture. Ainsi, après avoir connu un fort développement jusqu'aux années 2000, la croissance du chiffre d'affaires se ralentit voire même est négative en 2003 en raison d'une conjoncture morose. Depuis cette date et jusqu'en 2007, le chiffre d'affaires croît de nouveau sans retrouver toutefois le niveau de l'année 2000, pour ensuite s'effondrer en 2008 [18]. Malgré une croissance en 2009 et 2010, en 2011, il reste inférieur à celui de 1997.

Le développement de ces tourismes de court séjour est lié à des évolutions récentes dans les mobilités touristiques ${ }^{9}$ qui

\footnotetext{
6 Selon les observateurs (OMT, Atout France, CRT, OTCP, etc.), il inclut ou non une partie des déplacements individuels dits de voyage d'affaires. Le terme de tourisme d'affaires est lui-même discuté, notamment en faveur de l'acronyme anglais MICE (Meetings, Incentives, Conferences/Conventions, and Exhibitions/Events) ou de l'appellation meeting industry. Aussi, certains acteurs vont choisir la dénomination d'un secteur des rencontres et événements professionnels (CRT Ile-de-France par exemple).

${ }^{7}$ Qui correspondent à des réunions dont l'objectif est de motiver les employés et cadres d'une entreprise.

${ }^{8}$ Cependant, la crise financière et économique depuis 2008 a accru cette sensibilité au prix, pour des raisons économiques et d'image [18].

${ }^{9}$ En termes d'hypermobilité de multi-accessibilité, de pratiques multimodales/intermodales, de temporalité (temps réel, échange diffusion), de réduction de la planification des séjours, d'autonomisation des comportements, d'intégration des TIC $[11,19]$. Les responsables institutionnels n'ont pris la mesure de ces évolutions que très récemment à l'échelle urbaine elle-même [20].
}

sont, pour certaines d'entre elles, favorisées par des moyens de transport performants tels que les dessertes TGV.

Premièrement, une desserte TGV, en partie ou en totalité sur ligne à grande vitesse, permet d'offrir un service de transport plus rapide. Elle est plus compétitive pour des moyennes distances que l'avion dans la mesure où l'usage de ce dernier génère obligatoirement une rupture de charge en raison du déplacement entre l'aéroport et la ville. Aussi, la desserte TGV semble la plus appropriée, si la gare ainsi que les lieux de congrès et séminaires sont centraux, la ville compacte et les aménités touristiques localisées au cœur des villes et/ou facilement accessibles. La compacité de la ville et son irrigation par des réseaux de transport performants à partir de la gare permettent que le temps gagné dans le trajet en train ne soit pas perdu à l'arrivée. La desserte TGV en gare centrale présente ainsi des avantages certains dès lors que la chaîne de mobilité touristique dans son ensemble est prise en considération. En outre, dans le cas du tourisme d'affaires, la sensibilité à la qualité de la desserte et à la réduction des temps de parcours est très élevée en raison d'une valeur importante accordée au temps pour les déplacements professionnels [21].

Deuxièmement, la desserte TGV permet d'éviter la fatigue associée à la conduite d'une voiture, les encombrements et les difficultés de stationnement croissantes au cœur des villes.

Enfin, un déplacement en TGV est moins onéreux qu'un déplacement en voiture dès lors qu'il est effectué seul ou en couple.

Cette dynamisation des tourismes urbain et d'affaires ne peut cependant se réaliser que sous certaines conditions : la desserte doit être adaptée aux déplacements de week-end pour le tourisme urbain et de semaine pour le tourisme d'affaires. Un cadencement des allers-retours est ainsi important pour le tourisme d'affaires. Des aménités touristiques importantes $[5,6,22]$ et, pour le tourisme d'affaires, une infrastructure hôtelière conséquente en termes de qualité mais également de quantité, doivent être présentes, ce qui n'est pas toujours le cas dans les villes françaises.

Le développement général de ces tourismes a alors généré des attentes dans les villes françaises desservies par la grande vitesse ferroviaire.

\section{Des attentes en termes de tourisme urbain et d'affaires dans les grandes villes françaises}

Lors de la mise en place d'une desserte TGV, les villes nouvellement desservies ont des attentes importantes en termes de développement du tourisme urbain et d'affaires liées à l'attraction possible de la clientèle en provenance de l'Ile-de-France. 
Ainsi la mise en service de la Ligne à Grande Vitesse (LGV) Est-européenne en 2007, et les gains d'accessibilité qu'elle était supposée induire, devaient rendre le sillon lorrain plus attractif : «Une nouvelle clientèle (touristique) pourra être visée : francilienne en premier lieu, européenne ensuite, en provenance des autres régions françaises enfin. » [1]. Plus précisément, les villes de Metz, Nancy et Épinal [1] desservies par la LGV Est-européenne devaient pouvoir bénéficier d'une dynamisation du tourisme. Par exemple était espérée une dynamisation du tourisme de culture à Épinal (« images d'Épinal », musées, [2]). Des effets étaient également attendus en termes de tourisme d'affaires dans les villes du sillon lorrain [1] et notamment à Metz et Nancy [2].

De même, en 2005, un avis du CES de la région Alsace relevait " à un moment où l'activité touristique stagne, l'arrivée des TGV est une véritable opportunité pour renouveler l'offre touristique, en favorisant par exemple la venue de la clientèle francilienne » [23]. Les villes desservies par la LGV Rhin-Rhône en Franche Comté et en particulier Besançon qui est dotée d'un patrimoine architectural important, en espéraient tout autant [24].

Une dynamisation du tourisme est également attendue dans les villes qui seront desservies par la LGV Sud-ouest sur la ligne Bordeaux-Narbonne telles que Bordeaux et Toulouse [25].

Plus récemment encore, en Basse-Normandie, la quasitotalité des cahiers d'acteurs réalisés lors du débat public de la Ligne nouvelle Paris-Normandie (LNPN) évoque un développement du tourisme [26]. La Basse-Normandie étant un lieu de villégiature privilégié pour la clientèle parisienne, l'accessibilité renforcée à Paris induite par la mise en place de cette ligne nouvelle est supposée renforcer cette dynamique touristique. Ainsi à Caen, « en rapprochant Paris de la mer, les fonctionnalités offertes par la LNPN permettront d'optimiser le potentiel touristique balnéaire, mémoriel et patrimonial de l'agglomération caennaise et de son littoral. Le projet de ligne nouvelle permettra également de multiplier les courts séjours, notamment des Franciliens, et de favoriser l'émergence dans l'agglomération de manifestations à caractère culturel ou professionnel (congrès) » [27].

De même, dans le cadre du débat public de la LGV Paris-Orléans-Clermont-Ferrand-Lyon (POCL), Roanne voit dans une possible future desserte un outil au service du développement de ses tourismes notamment gastronomique et événementiel : « La desserte TGV de Roanne permettra de renforcer l'offre touristique de week-end, d'affaires et liée à l'événementiel, en se rapprochant des bassins de clientèle lyonnais, clermontois et parisien. Elle confortera également la fonction d'accueil de congrès » [28]. Le tourisme d'affaires est ainsi vraisemblablement celui qui est le plus impacté [25].
Paradoxalement, le territoire francilien et la façon dont il pourrait bénéficier de la grande vitesse ferroviaire, est peu étudié, au regard de la littérature (grise en particulier) produite sur les autres territoires desservis [29]. Les problématiques en Ile-de-France se sont longtemps focalisées sur le système, l'organisation et le fonctionnement du cœur du réseau. Les enjeux liés à la convergence des flux ferroviaires à grande vitesse sur le territoire, supposé $a$ priori bénéficiaire et bien desservi, et l'intensité des enjeux régionaux de transport, semblent reléguer la réflexion sur les impacts et enjeux franciliens de la grande vitesse au second $\operatorname{plan}^{10}$.

Ainsi, ces attentes dans les villes françaises laissent supposer que les effets de polarisation parfois évoqués au sujet des dessertes TGV n'existeraient pas dans le domaine du tourisme.

\section{Une possible polarisation associée aux dessertes ferroviaires à grande vitesse en matière de tourisme ?}

Avant la mise en service de la première ligne à grande vitesse entre Paris et Lyon en 1981, des craintes quant au renforcement de la centralité parisienne sont mises en avant [31,32]. Alain Bonnafous pointe ainsi «le sens dans lequel le TGV peut jouer : celui d'une centralité parisienne croissante » [31]. Au regard de différentes études $[32,33]$ suite au développement effectif des dessertes, le TGV apparaît comme un instrument de lecture des relations entre Paris et les villes françaises desservies, moins analysé comme un repositionnement de Paris mais bien comme un positionnement par rapport à Paris [32]. La région parisienne est alors un marché [32] et la question des effets est posée du point de vue des dessertes françaises.

La littérature relative aux effets de polarisation des dessertes ferroviaires à grande vitesse est cependant nuancée [34]. Certains auteurs suggèrent que l'arrivée d'une desserte TGV peut être associée au renforcement des grands pôles urbains desservis [31,35,36] ; pour d'autres, ce renforcement est différent selon les cas [37] voire marginal [38]. Enfin, dans certains cas, une desserte TGV peut bénéficier aux grandes villes intermédiaires [7].

Si le débat en termes de polarisation ne semble pas tranché, force est de constater qu'en termes de tourisme qu'il soit urbain ou d'affaires, la question n'est quasiment pas évoquée. Les effets des dessertes TGV sur le tourisme

\footnotetext{
${ }^{10}$ Cependant, le projet de nouveau schéma régional d'aménagement à partir de 2005, les contributions des différents acteurs de la région Ile-de-France, en 2005-2006, puis après 2011, les projets de Grand Paris, et les projets d'infrastructure ferroviaire à grande vitesse en Ile-de-France, Interconnexion Sud en particulier, [29,30] semblent indiquer une inflexion.
} 
n'intègrent pas la dimension « hiérarchie urbaine ». Si la théorie des places centrales, développée principalement par Christaller et Lösch explique la concentration des services de haut niveau dans les places centrales de rang élevé, elle ne s'applique pas aux services touristiques. En effet, Christaller relève : There is also a branch of economy that avoids central places and the agglomeration of industry. This is tourism. Tourism is drawn to the periphery of settlement district [39].

Pour autant, si de nombreux facteurs sont susceptibles de jouer un rôle dans l'attractivité des territoires en termes de tourisme (paysage, climat, patrimoines, etc.), force est de constater que metropolitan areas continue to dominate as principal centers of tourism activity [40].

Cependant, le tourisme reste toujours marginalisé au sein des recherches urbaines. D'une part, comme le souligne Stock, en 2012, il joue « un rôle marginal dans les recherches géographiques tentant de proposer des modèles qui visent à rendre intelligible les questions de l'urbain » [41]. D'autre part, la façon dont il pourrait bénéficier des dessertes TGV est relativement peu investie $[6,22]^{11}$. Le triptyque « TGV-Tourisme-Hiérarchie urbaine »n'est pas analysé.

Ainsi la question des interrelations entre TGV et tourisme dans la capitale semble s'être posée de façon marginale lors des premières mises en service comme lors de l'extension du réseau. Le développement des dessertes TGV n'a suscité que peu d'attentes en termes d'activités touristiques pour la destination Paris. Au regard de celles des acteurs dans les autres régions françaises, il apparaît surprenant que pour Paris, une des premières destinations touristiques mondiales, de surcroît cœur du réseau ferroviaire à grande vitesse français, ce lien n'ait pas été saisi ou du moins questionné. De par la centralisation politique et économique qui caractérise la France, la desserte TGV de Paris semble relever de l'évidence. Aucune politique globale de développement touristique associant les dessertes TGV n'a jamais émergé en Ile-de-France. Après le succès du TGV Paris-Lyon, le renforcement continu de la desserte francilienne n'a pas été questionné : ses impacts n'ont pas été estimés. Pourtant, les TGV vont dans les deux sens, et par conséquent essentiellement en direction de Paris. Or, si l'Ile-de-France est envisagée dans les stratégies de développement des villes françaises, c'est uniquement comme un bassin émetteur.

Cependant certaines attentes des acteurs concernant le développement touristique par la desserte TGV à Paris-Ile-de-France semblent poindre et renouvellent la lecture de la centralité.

\footnotetext{
${ }^{11}$ La session spéciale organisée sur la thématique « TGV, Tourismes et Territoires » au colloque de l'ASRDLF 2012 visait à combler ce manque.
}

\section{Dessertes TGV et tourisme urbain et d'affaires : la double centralité parisienne}

Le propos de cet article n'est ni de corréler le développement touristique parisien et le renforcement de sa desserte TGV, ni de l'opposer aux stratégies des villes accueillant un service à grande vitesse nouveau ou ayant considérablement amélioré leur niveau de desserte. Il s'agit de mettre en évidence un paradoxe et de questionner certaines idées reçues.

Alors que Paris est une destination touristique et le cœur du système TGV français, les attentes se sont concentrées dans les villes et métropoles régionales. Aussi, la mise en regard des dessertes permet à la fois d'illustrer et de qualifier le renforcement de la desserte parisienne, de comprendre le repositionnement à Paris à l'échelle nationale, mais aussi de fournir des hypothèses concernant le positionnement des acteurs franciliens sur les enjeux entre tourisme et dessertes TGV. L'importance de la destination touristique Paris détermine le contexte dans lequel les acteurs franciliens se saisissent de la question de l'accessibilité et de la territorialité de leur destination.

\section{Paris, la croissance des dessertes ferroviaires ou la centralité renforcée}

En France, la desserte, sur ligne dédiée ou sur ligne classique, s'est développée en axe autour de Paris, et, dans une moindre mesure, via des TGV «province-province». Mais ces derniers qui assurent les liaisons entre destinations françaises ${ }^{12}$ passent également par les gares franciliennes d'interconnexion ${ }^{13}$.

Cette organisation de la desserte prolonge le développement du réseau en étoile autour de Paris. Le réseau dédié s'est en effet développé en axe, puis en prolongement de ces axes, secondé par les lignes de rocade - contournement ou interconnexion - assurant les connexions entre ces différents axes (Table 1).

Paris et sa région sont ainsi desservis par la grande vitesse par quatre gares parisiennes ${ }^{14}$, auquel s'ajoute la Gare d'Austerlitz qui accueille notamment les trains Elipsos en

\footnotetext{
12 Cette desserte «province-province », aussi appelée intersecteur, assure, via les gares d'interconnexion franciliennes, les liaisons Nord Sud-est, Nord Atlantique, Atlantique Sud-est, Est Nord, Est Atlantique ou encore Normandie Sud-est, selon la terminologie SNCF.

${ }^{13}$ D'autres liaisons « province-province » existent et ont été renforcées par les récentes mises en service, en particulier le premier tronçon de la LGV Rhin-Rhône.

${ }^{14}$ Les gares parisiennes ont donc été aménagées pour accueillir les TGV et opérateurs à grande vitesse ferroviaire au fur et à mesure de l'extension du réseau : Gare de Lyon en 1981, puis Gare Montparnasse (1989-1990), gare du Nord (1993) et gare de l'Est en 2007. Dans le cas francilien, aux dessertes TGV s'ajoutent les dessertes européennes, principalement Eurostar, Thalys, Lyria et le premier concurrent de la SNCF en France, Thello, depuis décembre 2011.
} 


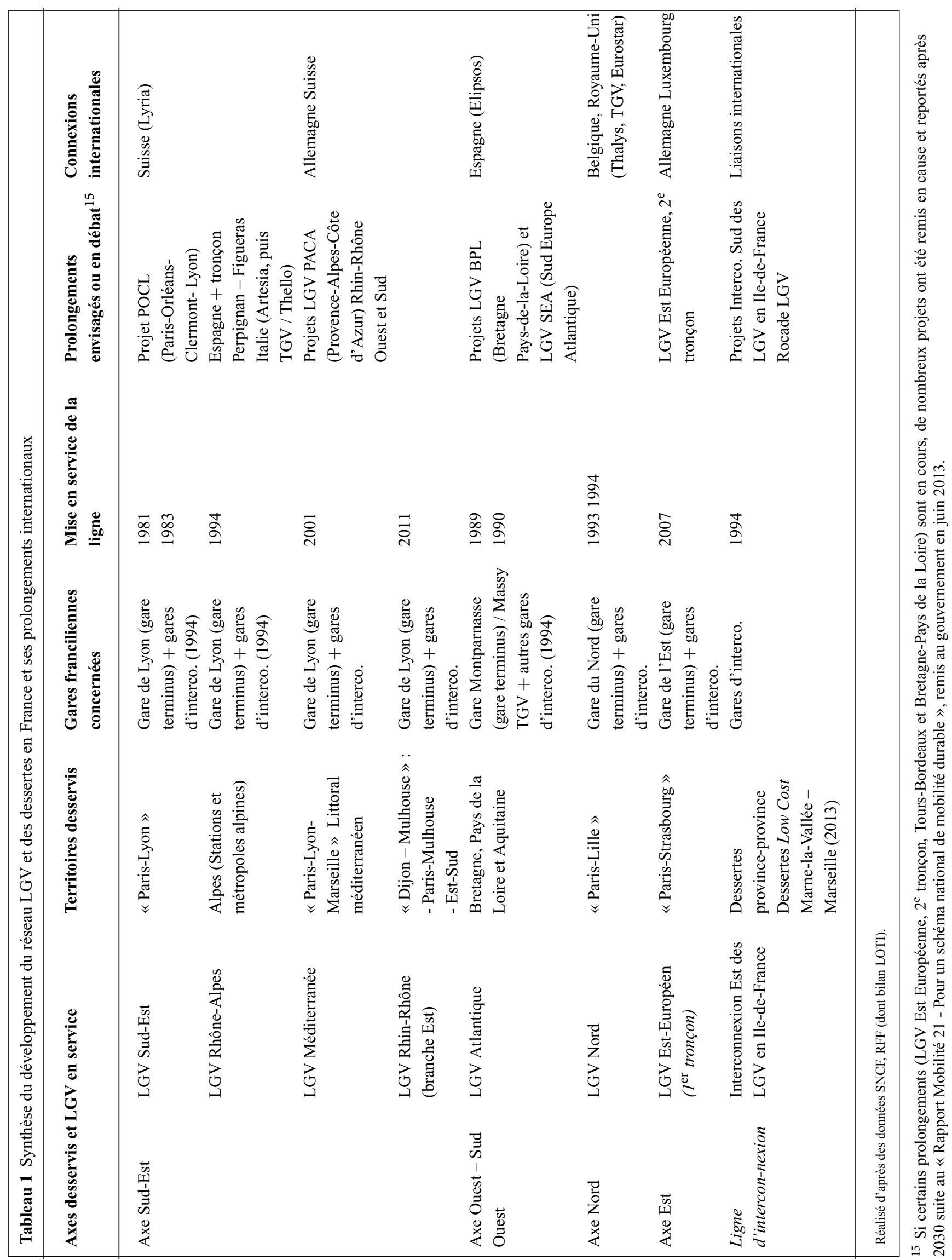

Necplus 
provenance d'Espagne, et par trois gares d'Interconnexion ${ }^{16}$ en petite et moyenne couronnes parisiennes ${ }^{17}$ : Massy TGV, Aéroport CDG TGV et Marne-la-Vallée Chessy.

Au-delà de leur localisation en périphérie métropolitaine de Paris, les gares divergent par leur type de desserte [30]. Les gares parisiennes, sont des gares terminus, chacune sur un axe : la desserte est donc monodirectionnelle ${ }^{18}$. Au contraire, les gares TGV d'Interconnexion sont multidirectionnelles.

Cette organisation des dessertes ferroviaires à grande vitesse met en évidence la centralité parisienne et son renforcement au cours des 30 dernières années ${ }^{19}$. En 2012, parmi les 186 gares françaises qui accueillent la grande vitesse ferroviaire, en dehors des gares et haltes franciliennes, seules 13 ne sont pas accessibles directement depuis Paris ou par l'une des gares franciliennes (Fig. 2).

Cette centralité renforcée à l'échelle nationale, et européenne, est cependant à mettre en perspective à l'échelle métropolitaine. À Paris-Ile-de-France, le trafic TGV a presque doublé en 10 ans, passant de 48 millions de voyageurs en 1999 à plus de 91,5 millions en 2009 [43, 44], alors que cette même année, 122 millions de voyageurs grande vitesse (TGV et international) sont transportés par la SNCF. Or, si le développement du réseau et des dessertes en région a un effet multiplicateur sur les dessertes de Paris-Ile-de-France, ce dernier s'opère de façon différenciée selon les territoires de la métropole [30,43].

Sur les 92,5 millions de voyageurs " grande vitesse » transitant en Ile-de-France en 2010, les gares TGV de Paris intra-muros concentrent $90 \%$ du trafic, avec en 2011, 30,5 millions de voyageurs TGV en gare de Lyon, 25,3 en gare Montparnasse, 19,9 en gare du Nord et 11,2 en gare de l'Est [43]. Les gares d'Interconnexion de CDG TGV (3,95 millions de voyageurs), MLV Chessy $(2,98)$ et Massy $(1,6)$ enregistrent toutefois une croissance soutenue depuis le milieu de la décennie $2000^{20}$ (Fig. 3).

D'ici 2020, la SNCF estime que le trafic voyageurs TGV devrait augmenter de $22 \%$ dans les gares intra-muros et

\footnotetext{
${ }^{16}$ Aussi appelées gares de jonction dans le cas francilien (SNCF), et associées aux dessertes TGV jonction (ou Intersecteur).

17 Ainsi que plusieurs « haltes » TGV : Juvisy, Mantes-la-Jolie, Massy Palaiseau et Versailles Chantiers (décembre 2011).

18 C'est pourquoi la SNCF considère les gares parisiennes comme équivalentes à une gare, chaque gare desservant uniquement un axe puisque les connexions entre les axes ne sont pas possibles [43].

${ }^{19}$ Seule la LGV Rhin-Rhône n'est à proprement parler pas centrée sur Paris, de façon radiale, ou en termes de prolongement. Cependant, si cet axe a permis le développement de nouvelles dessertes Intersecteurs, notamment entre le Sud de la France et l'Est du Pays ainsi qu'avec l'Allemagne, une grande partie de la desserte est organisée en lien avec Paris [42].

${ }^{20}$ Pour les 10 premiers mois 2011, la fréquentation augmente de $17 \%$ à MLV Chessy, $14 \%$ à Massy TGV et $5 \%$ à CDG TGV [43].
}

qu'il devrait doubler dans les gares d'interconnexion [43] $]^{21}$. Ainsi, la fréquentation augmente mais de façon différenciée selon la localisation de la gare, l'axe et le type de desserte.

Cette organisation de la desserte dans la métropole parisienne explique en partie les stratégies d'acteurs. Alors que dans les villes nouvellement reliées au réseau LGV, les acteurs se mobilisent parfois de façon collective autour du possible développement touristique lié à ces dessertes, les stratégies mises en place à Paris sont ponctuelles et localisées sur certains espaces de la métropole parisienne.

En Ile-de-France, les acteurs se sont posés dès la fin des années 1980 la question des enjeux entre tourisme et desserte TGV pour le cas précis de la gare de Marnela-Vallée TGV et de la desserte des parcs Disneyland $\AA$ Paris [36,45]. Mais l'imbrication bien spécifique des enjeux de dessertes à grande vitesse ferroviaire et touristiques sur ce territoire constitue à la fois une référence et un contre exemple pour d'autres projets [46 ${ }^{22}$. La polarisation touristique est en effet en partie indépendante du secteur de Disney dans la destination Paris [12], ce qui renforce la spécificité des enjeux ferroviaires et touristiques qui lui sont associés.

À l'échelle de la destination, la qualité de son accessibilité est vantée en France et en Europe. La desserte TGV devient un objet marketing. Plusieurs campagnes ciblées ponctuelles dans le cas de dynamiques de projet ou avec un investissement en pointillé dans le temps ont ainsi été mises en place. C'est le cas avec l'arrivée du TGV, de l'ouverture d'un bureau de l'Office du Tourisme dans la Gare de Lyon en 1981, puis à la gare du Nord, mais ce partenariat avec la SNCF prend fin en 1997. La campagne britannique de l'Office du tourisme et des congrès de Paris (OTCP) en 1995 est un autre exemple [47].

Mais globalement, les attentes identifiées dans les territoires nouvellement desservis ne se retrouvent pas à l'échelle de la destination, et les enjeux conjoints des dessertes ferroviaires à grande vitesse et les enjeux touristiques ne sont pas analysés.

À Paris, destination touristique de rang mondial, les acteurs n'ont pas les mêmes problématiques de développement que d'autres destinations. Pourtant, du fait de l'importance de Paris comme destination touristique, il est étonnant que la région capitale ne soit envisagée que comme

\footnotetext{
21 La mise en service en avril 2013 des TGV low cost à la gare de Chessy-Marne-la-Vallée ( $c f$. infra) devrait contribuer à cette croissance.

${ }^{22}$ C'est le cas de Versailles par exemple [29]. Pour autant, au regard des attentes d'autres destinations françaises, les attentes ne semblent pas illégitimes.
} 


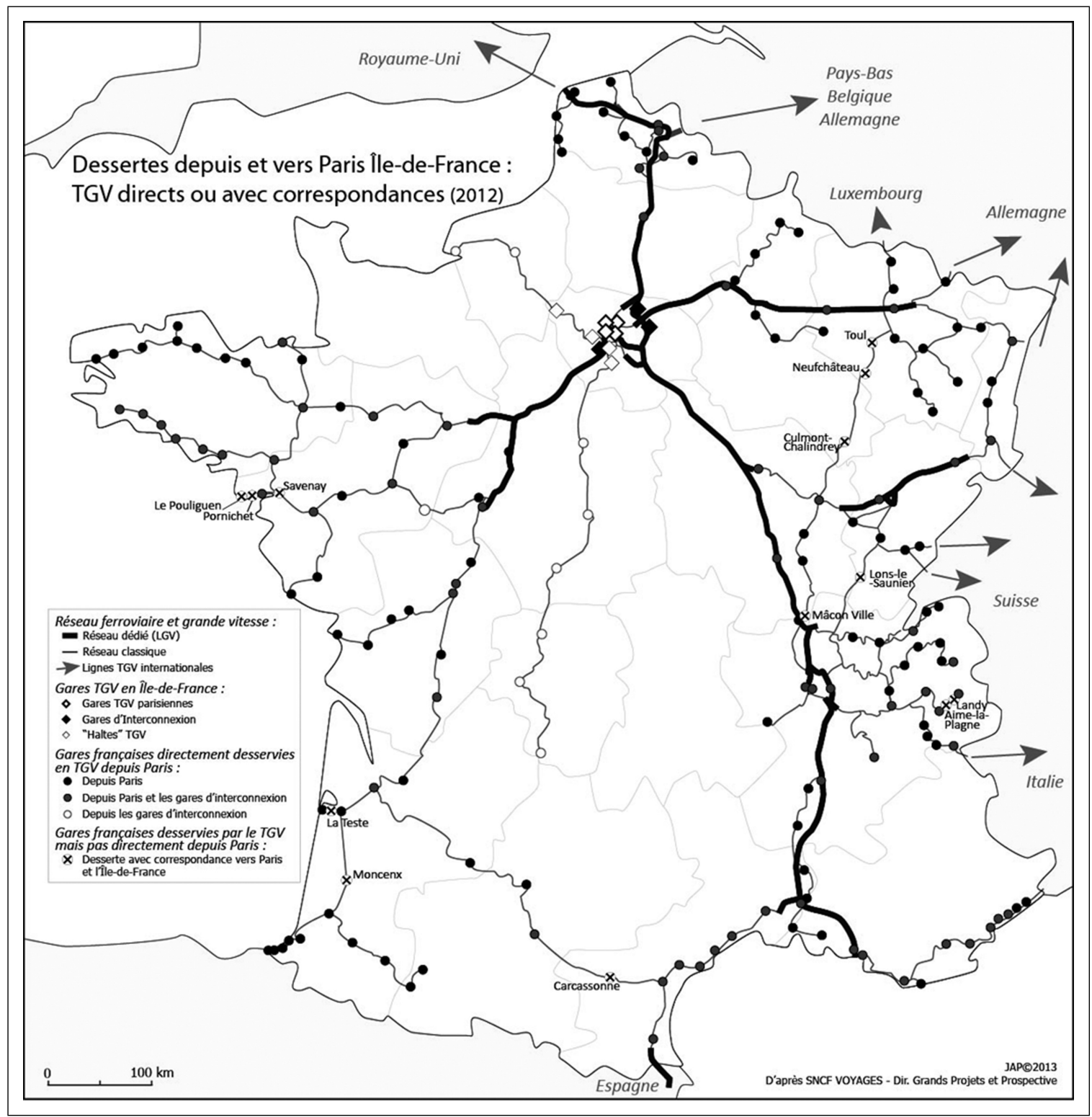

Fig. 2 Les villes desservies par TGV directement ou en correspondance au départ et à destination des gares d'Ile-de-France en $2012^{23}$

un bassin émetteur de touristes. De plus, l'intensité et l'évolution des flux touristiques au sein de la métropole parisienne renouvellent l'intérêt porté à son accessibilité, dans un contexte régional de débat intense sur la mobilité.

${ }^{23}$ Cette carte met en avant plusieurs niveaux d'accessibilité depuis Paris-Ile-de-France : les gares accessibles depuis les gares parisiennes et franciliennes d'interconnexion, et celles qui le sont uniquement depuis les gares parisiennes ou les gares franciliennes. Les quelques villes mentionnées sur la carte ne sont pas desservies directement par TGV au départ de l'Ile-de-France mais en correspondance.

\section{Destination touristique et accessibilité : le renforcement de la centralité touristique?}

Si Paris concentre les dessertes, elle est également une destination touristique majeure pour les touristes français comme internationaux et notamment européens de proximité, particulièrement en termes de tourisme d'affaires. Mais, les acteurs en charge du tourisme à Paris Ile-de-France sont confrontés à une concurrence accrue entre les destinations touristiques et à l'évolution des pratiques. Associée aux enjeux actuels d'accessibilité, cette situation conduit à un renouvellement de leur regard sur le lien entre tourisme et desserte TGV et ses potentiels. 


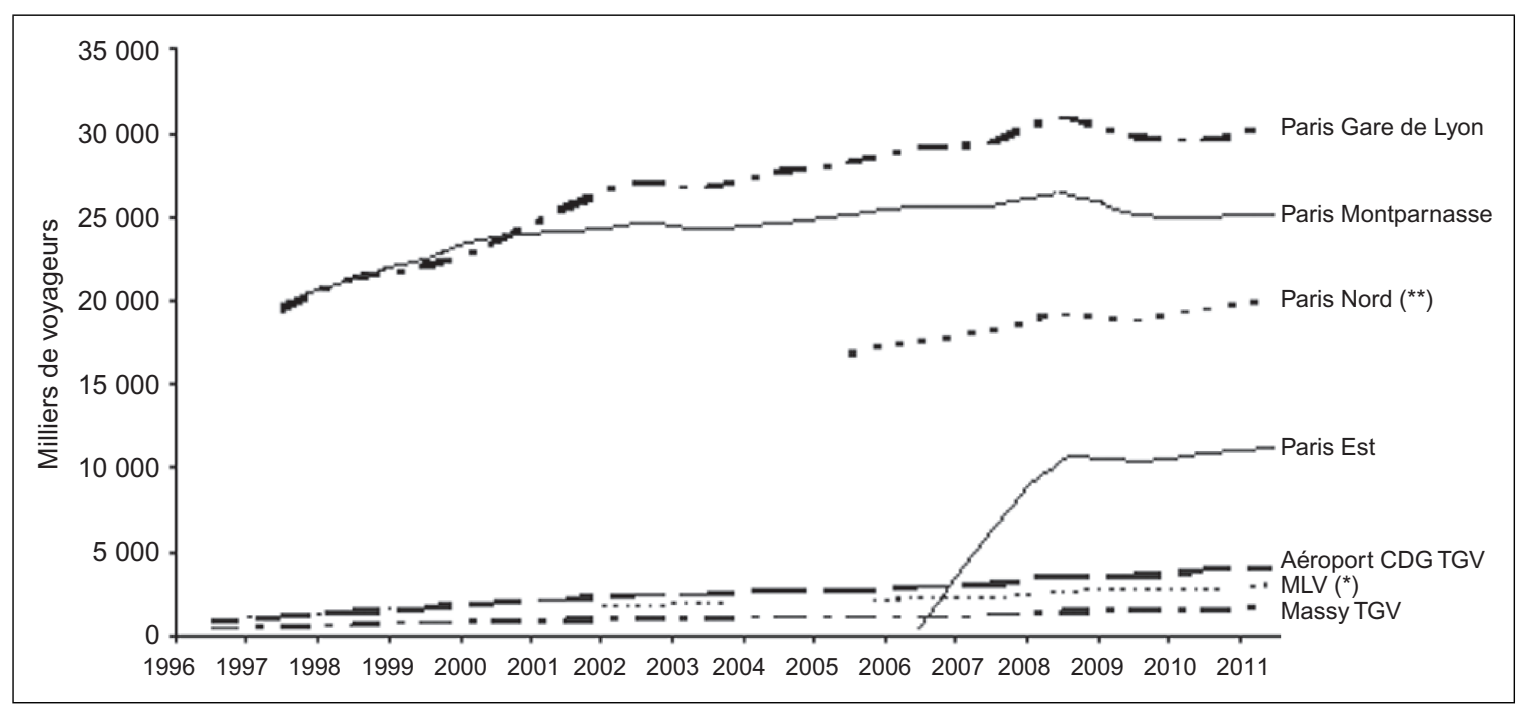

Fig. 3 Évolutions de la fréquentation des gares TGV à Paris intra-muros et des gares TGV franciliennes d'interconnexion entre 1996 et 2011

Réalisé par les auteurs, données SNCF

(*) Fréquentation Eurostar comprise

(**) Fréquentation Eurostar et Thalys comprises (données non disponibles avant 2005)

\section{Une destination majeure, qui dépasse la seule destination « Paris »}

Paris concentre des aménités touristiques importantes du point de vue du tourisme urbain et ses infrastructures en termes de tourisme d'affaires la placent au tout premier plan des villes françaises. Elle concentre également les sièges sociaux qui sont les principaux prescripteurs de tourisme d'affaires $[48]^{24}$.

Le patrimoine comme les infrastructures d'accueil et d'accès de Paris-Ile-de-France en font la première destination touristique mondiale en termes de fréquentation et la première destination touristique française avec la plus forte fréquentation touristique ${ }^{25}$ des régions françaises [50]. Qu'il s'agisse de tourisme urbain ou d'affaires, la destination Paris est particulièrement attractive pour la clientèle française et européenne.

En 2010, elle totalise respectivement 33,8 millions de nuitées hôtelières pour les visiteurs internationaux et 32 millions de nuitées hôtelières pour les résidents français [49]. Les touristes européens représentent plus de $62 \%$ des arrivées hôtelières à Paris en $2010^{26}$ et avec la clientèle

\footnotetext{
${ }^{24}$ Paris concentre 37 des 39 sièges sociaux localisés en France des 500 entreprises du classement Fortune Global dans le monde.

${ }^{25}$ Tous types de tourisme (agrément et affaires) et origines des visiteurs (internationale et française) confondus. En ce qui concerne l'accueil des visiteurs français, elle est en deuxième position avec près de $10 \%$ des voyages effectués en France [49].

26 Il s'agit principalement des britanniques, espagnols, italiens, allemands et visiteurs Benelux [49].
}

française, 84,2 \% des arrivées en 2010 [49], une situation renforcée par des évolutions structurelles - court séjour, partition des vacances etc. - et la conjoncture économique. Le tourisme urbain en Ile-de-France, en particulier de la clientèle française, s'est développé de façon importante depuis les années $1970^{27}$. À Paris même, les arrivées de la clientèle de tourisme urbain ont triplé entre 1970 et 2010[47].

Ces tendances générales sont encore plus marquées pour le tourisme d'affaires qui occupe une place importante dans l'activité touristique de la région, et représente $46 \%$ des nuitées hôtelières en 2010 [49]. Ainsi, sur le plan national, concernant l'ensemble des activités de tourisme d'affaires, le déséquilibre en faveur de l'Ile-de-France est fort, avec une concentration des deux tiers de ces activités et de $80 \%$ des activités de congrès et d'exposition en France [51]. À Paris, la décennie 1970 est marquée par le développement d'infrastructures d'accueil liées au tourisme d'affaires, avec en particulier l'ouverture de Palais des Congrès en 1974, associé au développement d'hôtel de grandes chaînes entre 1972 et 1978. La décennie 1980 marque une consolidation générale de l'offre hôtelière et de sa répartition $[47]^{28}$.

\footnotetext{
27 L'Office du tourisme et des congrès de Paris note ainsi le développement des courts séjours urbains, en lien avec les évolutions de la société (congés payés, 35h) [47] et le développement de l'accessibilité, en particulier ferroviaire.

${ }^{28}$ L'offre hôtelière en particulier enregistre son plus fort développement (en chambre et en établissement), avec une montée en gamme
} 


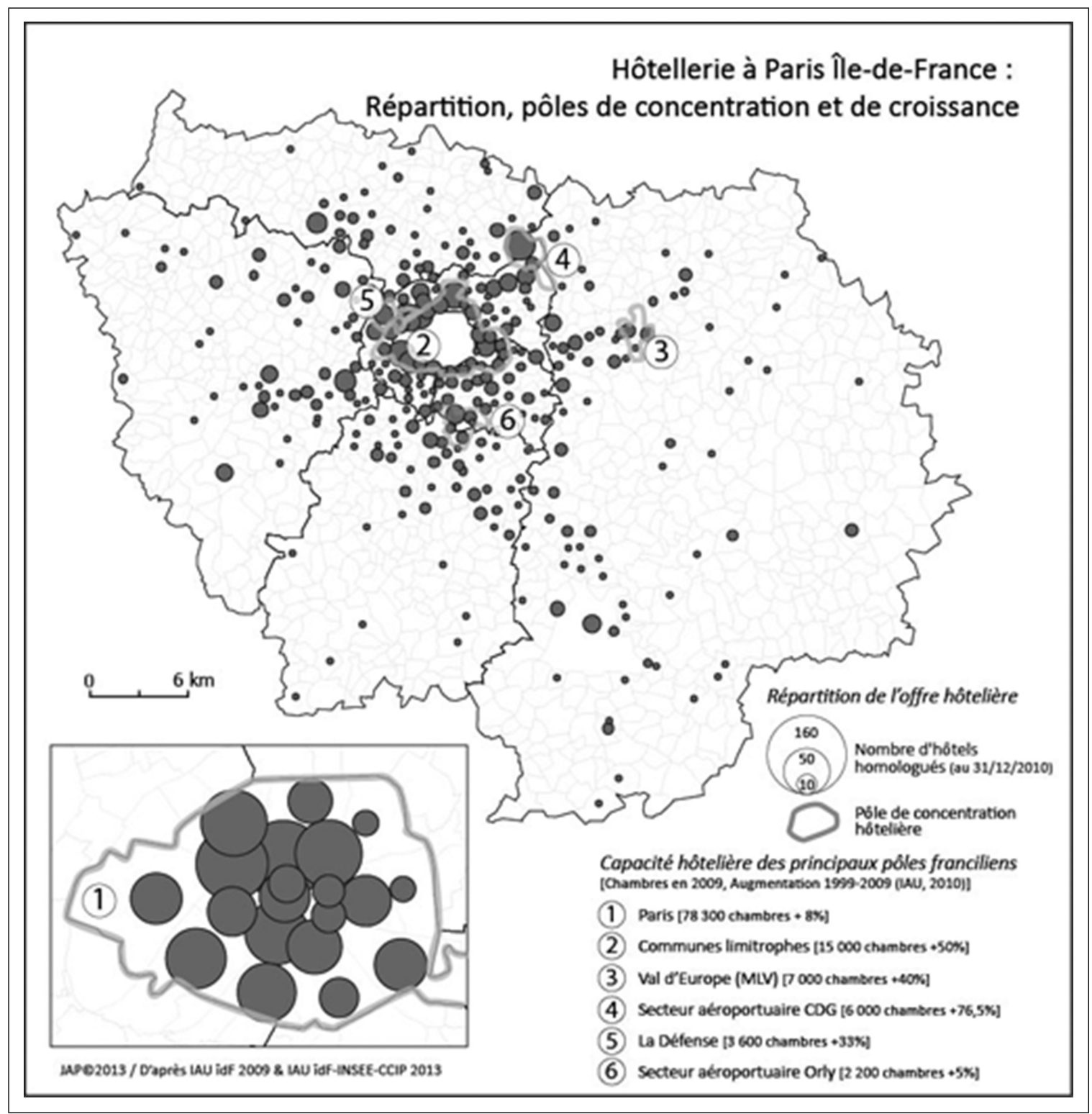

Fig. 4 Évolution et répartition de l'offre hôtelière à Paris-Ile-de-France

Cependant, si la destination Paris possède un tel rayonnement, c'est aussi en partie au titre de Paris Ile-de-France, selon un processus amorcé au tournant des années 1970 et 1980 . Les territoires de cette destination ${ }^{29}$ sont en effet

notable (3 étoiles), qui est confirmée au cours des années 1990 (4 étoiles).

${ }^{29}$ Les concertations entre acteurs institutionnels et privés dans le cadre de la stratégie régionale 2011-2016 [52], ont mis en avant la question $\mathrm{du} /$ des territoire(s) de la destination (et les enjeux de gouvernance associés) : zone dense (Paris et la petite couronne) ? « Métropole » (laquelle) ? Région ? Bassin parisien ? Cependant, les acteurs de la région se sont accordés pour la première fois sur la puissance de la marque « Paris » et son rayonnement sur l'ensemble de la destination. Depuis 2012, une stratégie de marque territoriale est en cours d'élaboration, pilotée par la Région Ile-de-France. multiples : destination urbaine, métropolitaine, régionale. Paris est une marque particulièrement puissante dans le monde entier $[47,49]$ et la destination ne s'arrête pas au périphérique. "Pour les touristes, ce "grand Paris" est déjà une réalité " écrit l'OTCP en conclusion de son bilan sur 40 ans de tourisme à Paris [47]. Une destination touristique ne repose en effet pas sur les découpages institutionnels.

En 2010, la répartition des nuitées hôtelières entre Paris et le reste de l'Ile-de-France est de $55 \% / 45 \%$ [49,52]. Pour les seuls visiteurs français, l'Ile-de-France hors Paris recense $60 \%$ des arrivées [47,49]. C'est ainsi en Ile-de-France, en dehors de Paris intra-muros (Fig. 4) que la croissance du parc hôtelier est la plus forte. 
En effet, à partir du milieu des années 1980, l'offre se développe dans un premier temps en petite couronne, le « Paris du métro », puis autour de grands pôles tels que le secteur de Villepinte (tourisme d'affaires), Val d'Europe (Parcs Disneyland $®$ Paris) et plus récemment les secteurs de Roissy (aéroport Charles-De-Gaulle), la Défense (pôle économique) ou encore Orly (zone aéroportuaire). Au cours des dix dernières années, la tendance s'est confirmée, avec une croissance du parc autour des pôles existants $(+18,8 \%)$, la part du pôle parisien étant en diminution au profit des pôles périphériques (+33\%) [52]. L'offre atteint 150000 chambres pour plus de 2300 établissements [44].

Cette répartition de l'offre hôtelière s'inscrit dans la double dynamique de concentration et d'extension de l'espace parisien [12]. À la polarité de l'hypercentre touristique parisien autour de la Seine (Central Tourism District, [12]), sont juxtaposées des pratiques duales de l'espace, avec les satellites du centre (Montmartre, la Défense etc.) et quelques grands pôles périphériques (Val d'Europe (Disneyland $($ Paris), Versailles, Roissy) (Fig. 4). De même, les pôles du tourisme d'affaires fortement liés aux capacités d'accueil, sont concentrés autour de Paris et la petite couronne, auxquels s'ajoutent les grands pôles de Villepinte et Disneyland $\left(\right.$ Paris $^{30}$.

L'analyse des répartitions hôtelières à Paris-Ile-de-France permet ainsi de lire les pratiques et répartitions touristiques dans la destination. Les principales concentrations hôtelières se situent ainsi à proximité des grands pôles d'accès à la métropole et les six premiers pôles hôteliers de Paris-Ile-deFrance, sont soit desservis par une (des) gare(s) TGV soit dans le secteur d'un projet de gare ${ }^{31}$ (Fig. 4).

Pour autant, si la localisation et les choix d'hébergements hôteliers notamment s'éloignent du cour touristique, ceux-ci ne traduisent pas une métropolisation touristique effective, mais un processus en cours, encouragé par les acteurs institutionnels en charge du tourisme [44,52,53]. C'est bien Paris que les touristes viennent visiter comme la forte concentration et la centralité des pratiques touristiques dans l'espace en attestent, mais des pôles touristiques secondaires métropolitains secondent ces flux [12], renforcés par les pratiques et attentes des repeaters [52]. Dès lors, espace central, espace parisien et espace francilien s'imbriquent et se lisent selon leur fréquentation et les déplacements associés : à pied (centre), le «Paris du métro », et celui du RER [12]. C'est à l'échelle de la destination métropolitaine

\footnotetext{
$\overline{30 \text { Disneyland } ®}$ Paris est également le premier site intégré européen de tourisme d'affaires, avec plus de 1000 événements organisés par an ( $C f$. communication en ligne sur le site de Disney Business Solutions, actualisé en 2012).

${ }^{31}$ La gare d'Orly est associée au projet d'Interconnexion Sud des LGV en Ile-de-France ; à un horizon plus lointain, le projet de rocade LGV en Ile-de-France suggère l'implantation d'un gare TGV nouvelle dans le secteur de Nanterre-La Défense [43].
}

Paris-Ile-de-France que les enjeux touristiques se déclinent désormais.

Ainsi, en novembre 2011, la région Ile-de-France adopte la Stratégie régionale de développement du tourisme et des loisirs (SRDTL) 2011-2016. Le classement de la destination au niveau mondial et son maintien constituent un enjeu pour les acteurs institutionnels. Selon les projections d'arrivées touristiques et en fonction de l'importance des politiques mises en place [52], 5 à 10 millions d'arrivées supplémentaires devraient être enregistrées d'ici 2020, conduisant à une augmentation des nuitées notamment hôtelières. Or, en particulier dans le centre touristique, la question de la saturation est déjà un enjeu. $\mathrm{La}$ fréquentation touristique intense génère des conflits d'usages de l'espace, en particulier dans l'hypercentre $[12,47]$. La métropolisation des pratiques touristiques est la réponse avancée par les acteurs institutionnels de la région. $\mathrm{La}$ consolidation de polarités périphériques permettrait de répondre à la saturation progressive des sites majeurs et participerait au renouvellement de l'offre touristique [52]. La métropole touristique apparaît comme un enjeu pour les acteurs du tourisme en termes de gouvernance [53], d'inscription dans les dynamiques territoriales en cours (Grand Paris, Paris métropole) mais aussi pour acter et développer le timide processus de métropolisation des pratiques touristiques en cours. Ce faisant, elle modifie la façon dont les acteurs du tourisme appréhendent la question de l'accessibilité et des dessertes TGV.

\section{Enjeux touristiques métropolitains et regards renouvelés sur les dessertes TGV}

La métropolisation du tourisme affichée par les acteurs en charge du développement touristique est directement associée aux enjeux d'accessibilité [52]. Argument marketing, l'accessibilité était avant tout envisagée à l'échelle de la métropole. Or, de par les orientations choisies dans le cadre des travaux préparatifs à la nouvelle stratégie régionale, la question de l'accessibilité s'est déplacée vers celle des territoires de la métropole $[44,52]$. Cet enjeu, associé à la volonté de renforcer le processus de métropolisation autour des pôles périphériques apporte un éclairage nouveau aux dessertes de ces pôles, en particulier par les gares TGV d'Interconnexion.

En effet, désormais, l'enjeu porte autant sur l'accessibilité des sites touristiques en dehors de l'espace central de la métropole que sur celle des pôles permettant de desservir la métropole, et ce tant pour le tourisme urbain que le tourisme d'affaires $[44,51,53]$. Dans ce contexte, dans les stratégies actuellement développées, le TGV est identifié comme essentiel dans le développement de la destination 
Paris-Ile-de-France. Il passe alors d'un argument marketing à un outil potentiel de développement du tourisme ${ }^{32}$.

Les acteurs institutionnels franciliens du tourisme lisent le lien entre tourisme et TGV a posteriori comme un facteur très important. Ainsi, l'OTCP, souligne que les dessertes à grande vitesse sont des «éléments structurants » du tourisme pour la destination Paris [47]. Pour l'Institut d'aménagement et d'urbanisme (IAU) d'Ile-de-France, c'est l'augmentation de la fréquentation TGV en Ile-de-France et son doublement au cours de la décennie 2010 qui révèlent l'enjeu de la desserte pour l'activité touristique [44]. Signe de cette importance récente, depuis 2008, le CRT a mis en place un dispositif d'enquête permanent dans les trains à grande vitesse au départ de Paris et à destination de la France et de l'Europe, sur le modèle de son enquête sur les touristes venus en avion [49]. Outre l'estimation des flux touristiques $\mathrm{TGV}$, cette enquête permet pour la première fois d'identifier qui sont les touristes venus en TGV, leur provenance, leurs motivations et leurs pratiques par rapport à des touristes utilisant d'autres modes de transport ${ }^{33}$. De même, toujours en 2008, et 10 ans après la fermeture des bureaux de l'OTCP dans les gares, le Comité régional du tourisme réinvestit les gares en termes de structure d'accueil des touristes.

Deux éléments se distinguent toutefois fortement des attentes observées dans les métropoles régionales. Les acteurs en charge du tourisme et/ou porteurs de stratégie ont des difficultés à s'organiser [52,54]. La gouvernance est un enjeu fort. De plus, ils sont peu impliqués dans les projets en cours, et le regard nouveau qu'ils portent sur le potentiel de la desserte TGV est orienté vers les gares et dessertes existantes. Aussi, plutôt déconnectées des dynamiques de projet, les attentes ainsi identifiées ne s'intègrent pas dans les mêmes temporalités que celles constatées dans les autres villes françaises Par exemple, les enquêtes du CRT ne s'inscrivent pas, comme dans le cas des dessertes dans d'autres régions françaises, dans une démarche associant aménageurs (et transporteurs) dans une volonté de développement territorial. Le choix des gares enquêtées se concentre sur les gares parisiennes et celle de Marne-la-Vallée Chessy pour des raisons touristiques. Les différences de dessertes n'ont pas été identifiées, les projets de nouvelles gares TGV ne sont pas bien connus des acteurs institutionnels du tourisme. Ainsi, le Comité régional du tourisme n'est pas impliqué dans le projet d'Interconnexion

\footnotetext{
32 Le financement CIFRE d'une thèse portée par le Comité départemental du tourisme (CDT) de Seine-et-Marne ( $c f$. supra) illustre bien ce glissement. Le CDT s'interroge en effet sur le potentiel de la desserte à grande vitesse ferroviaire sur le développement du tourisme d'affaires, un axe identifié comme stratégique par les élus locaux et régionaux.

33 En 2010, sont concernés 10,3 millions de séjour soit plus de 33 millions de nuitées (à $56 \%$ hôtelières). Près d'un tiers des touristes TGV en Ile-de-France est européen [49].
}

$\mathrm{Sud}^{34}$. De même les aménageurs, côté transport, en charge de ces grands projets d'infrastructure qui sont à l'étude, n'identifient pas la dimension touristique. Concernant le développement de nouvelles gares, Marne-la-Vallée Chessy apparaît comme un référent écrasant ${ }^{35}$ mais il ne peut y avoir qu'un Disneyland en Europe ${ }^{36}$. Aussi, pour les acteurs en charge des projets TGV, en dehors de la desserte de Paris et des parcs Disney, le potentiel touristique semble faible.

Cependant, les réflexions sur les dessertes franciliennes, notamment dans le cadre des projets transport et grande vitesse, se sont considérablement renouvelées en particulier au cours de la décennie 2000 [30]. La possibilité et les potentialités d'implantation de nouvelles gares TGV en Ile-de-France ont abouti aux propositions d'évolution du modèle de desserte des gares d'interconnexion $[43,55]$. La multiplication des points d'accès en Ile-de-France permettrait le développement de TGV au départ des gares d'interconnexion franciliennes. Il n'est ainsi pas anodin que la SNCF ait choisi la gare de Marne-la-Vallée Chessy justement pour proposer sa nouvelle offre low cost sur l'axe sud. Côté Paris Ile-de-France, c'est en effet la force de la destination touristique qui appuie cette nouvelle offre de desserte.

\section{Conclusion}

Cet article se proposait d'investir la contradiction existant entre des attentes importantes en termes de développement des tourismes urbain et d'affaires dans les villes desservies par la grande vitesse ferroviaire, alors même que le développement de ces dessertes conduit à une polarisation sur Paris Ile-de-France, et que dans cette destination, elle-même touristique, ces attentes sont peu développées. Comme le soulignait Christaller [39], les analyses en termes de hiérarchie urbaine ne seraient pas pertinentes en matière de tourisme.

Ainsi, ces nouvelles dessertes suscitent des attentes nombreuses de la part des acteurs économiques des territoires concernés en termes de développement des tourismes urbain et d'affaires. Mais ces attentes reposent

\footnotetext{
34 Sur ce projet, à l'échelle plus locale, le projet d'une gare TGV nouvelle à Lieusaint (77) soutenu par les acteurs seine-et-marnais et sud-francilien a éveillé l'intérêt de Seine-et-Marne Tourisme, sans pour autant que le tourisme ait été un axe de développement potentiel promu à l'échelle du projet.

35 Echanges dans le cadre des projets Interconnexion Sud et études Rocades TGV (2009-2012) [29,30].

${ }^{36} \mathrm{Si}$ en 2011, la fréquence de la gare TGV de Marne-la-Vallée Chessy atteint 3 millions de voyageurs, l'enquête du CRT 2010 révèle que $66 \%$ des voyageurs au départ de cette gare ont visité les parcs. Les parcs ont attiré 15,7 millions de visiteurs en 2011 à $49 \%$ français. La part de visiteurs internationaux est élevée, et $5 \%$ des touristes étrangers en France ont visité le parc en 2010 (données Disney, Observatoire du tourisme 77, 2012).
} 
systématiquement sur la captation des touristes franciliens. La desserte n'est envisagée qu'en direction de la province : la région capitale est perçue uniquement comme un bassin émetteur.

Cette lecture « à sens unique "n'envisage pas le renforcement continu de la desserte de Paris-Ile-de-France et donc de la destination touristique parisienne, alors même qu'en matière de tourisme urbain et d'affaires, cette destination est particulièrement attractive pour les clientèles étrangères mais également pour les visiteurs français et que l'Ile-de-France concentre les activités de tourisme d'affaires. Ainsi, Paris-Ile-de-France, cœur du système ferroviaire à grande vitesse et destination touristique majeure apparaît à la fois comme une desserte renforcée et comme une destination concentrant différents flux touristiques. Au regard des attentes des villes nouvellement desservies en termes de développement touristique, le cas de Paris semble paradoxal. De surcroît alors que ces dessertes TGV contribuent à renforcer le cœur du réseau, Paris, la question de leur valorisation touristique au sein de la destination parisienne n'a pas été abordée. Cette relative absence peut s'expliquer autant par la structure du réseau et la dualité de la desserte que par les questions de gouvernance touristique dans la région, et plus largement avec les autres acteurs locaux. La centralité parisienne, renforcée par les nouvelles dessertes et l'attractivité de la destination touristique doivent être lues à l'échelle des territoires de la métropole touristique.

Mais la réflexion évolue aujourd'hui. La question des fonctionnalités franciliennes de la grande vitesse ferroviaire est intégrée aux réflexions qui animent les débats sur l'aménagement du territoire métropolitain, conduisant à s'interroger sur sa possible valorisation touristique. Elle serait alors un outil de diffusion du tourisme au sein de la métropole. Pour autant il reste à faire dialoguer entre eux les acteurs du tourisme francilien et les opérateurs ferroviaires.

\section{Bibliographie}

1. Ingénierie du Trafic et des Systèmes d'exploitation (ISIS) (2004) Analyse de l'impact du TGV-Est sur les agglomérations de Metz, Nancy, Épinal et Thionville. Rapport pour l'Agence de Développement des Investissements Extérieurs en Lorraine (ADIELOR) $58 \mathrm{p}$.

2. ISIS (2004) Analyse de l'impact du TGV-Est sur les agglomérations de Metz, Nancy, Épinal et Thionville. Rapport de phase 3 pour ADIELOR, $81 \mathrm{p}$.

3. Amiard D (1997) Le tourisme d'affaire et de Congrès dans l'agglomération mancelle In: Chevalier J Le Mans 6 ans après l'arrivée $d u T G V$. Groupe de recherche en géographie sociale, ESO - Espaces géographiques et Sociétés, Université du Maine, Le Mans, pp 51-67.

4. Bazin S, Beckerich C, Delaplace M (2010) Desserte ferroviaire à grande vitesse, activation des ressources spécifiques et développement du tourisme : le cas de l'agglomération rémoise. Belgeo 1-2: $65-77$.
5. Mannone V (1995) L'impact régional du TGV sud-est. Thèse pour l'obtention du doctorat de géographie, 2 tomes, Université de Provence Aix-Marseille I, $567 \mathrm{p}$.

6. Masson S, Petiot R (2009) Can the High Speed Rail Reinforce Tourism Attractiveness? The Case of the High Speed Rail between Perpignan (France) and Barcelona (Spain). Technovation 29: 611-617.

7. Urena JM, Menerault P, Garmienda M (2009) The High-Speed Rail Challenge for Big Intermediate Cities: a National, Regional and Local Perspective. Cities 26(5): 266-79.

8. Bazin S, Beckerich C, Delaplace M (2006) Analyse prospective des impacts de la Ligne Grande Vitesse Est-Européenne dans l'agglomération rémoise et en région Champagne-Ardenne. Rapport final remis à la région Champagne-Ardenne, $495 \mathrm{p}$.

9. Judd DR, Fainstein SS (1999) The Tourist City. Yale University Press, $352 \mathrm{p}$.

10. Maitland R (2009) National capital and city tourism. In: Maitland R, Ritchie BW. City tourism: National Capital Perspectives: 1-13.

11. Marzloff B, Bannwarth C (2005) Mobilité touristique et terminaux nomades : comment développer de nouveaux services? Pour la Direction du tourisme, $55 \mathrm{p}$.

12. Duhamel Ph, Knafou R (2007) Le tourisme dans la centralité parisienne. In: Saint-Julien Th, Le Goix R (dir.) La métropole parisienne. Centralités, inégalités, Proximités. Belin, pp. 39-64.

13. Duhamel Ph, Knafou R (2007) Les mondes urbains du tourisme. Belin, $360 \mathrm{p}$.

14. Kadri B (2007) La ville et le tourisme : relation ancienne, complexité nouvelle et défi conceptuel. Théoris 26(3): 76-79.

15. Vighetti JB (1994) Qu'est-ce que le tourisme urbain ? Cahier Espaces, Espaces tourisme \& loisirs 39: 31-35.

16. Direction Générale de la Compétitivité, de l'Industrie et des Services (DGCIS) (2012) Mémento du Tourisme 2011. Sous-direction de la Prospective des Études économiques et de l'Évaluation (P3E), $152 \mathrm{p}$

17. Direction du tourisme (2008) Le tourisme urbain dans l'hôtellerie française, un poids de plus en plus important. DGCIS, Tourisme Infos Stat $6: 12 \mathrm{p}$.

18. Coach Omnium (2012) MICE 2012 - Les entreprises implantées en France et le tourisme d'affaires. Etude annuelle, $20^{\mathrm{e}}$ édition, $58 \mathrm{p}$.

19. Desvignes C (dir.) (2009) Nouvelles mobilités touristiques. Les Cahiers Espaces 100, 124 p.

20. Atout France (2012) Faciliter les mobilités pour enrichir l'expérience touristique. Collection Rendez-vous en ville, Edition Atout France, $168 \mathrm{p}$.

21. Hammadou H, Jayet H (2002) La valeur du temps pour les déplacements à longue distance : une évaluation sur données françaises. Les Cahiers Scientifiques du Transport 42: 3-23.

22. Bazin S, Beckerich C, Delaplace M (2011) High speed railway, service innovations and urban and business tourisms development. In Sarmento M., Matias A. Economics and Management of Tourism: Trends and Recent Developments. Collecçao Manuais, Universidade Luisiada Editora, Lisboa.

23. Conseil Economique et Social d'Alsace (CESA) (2005) La stratégie régionale du tourisme en Alsace. Région Alsace, $12 \mathrm{p}$.

24. Villes et Régions Européennes de la Grande Vitesse (2009) 25 ans de la Grande Vitesse sur nos territoires, Cahiers de la Grande Vitesse. Numéro spécial.

25. Setec ORGANISATION (2005) Etude des effets de la réalisation d'une LGV entre Bordeaux et Narbonne sur le développement des aires urbaines d'Agen et de Montauban et sur l'aménagement des territoires traversés. Pour Réseau Ferré de France, 20 p.

26. Guinguand C (2012) La Ligne Nouvelle Paris Normandie, Quelles stratégies d'acteurs sur le territoire de la Basse-Normandie? Mémoire de Master 1, Université de Paris-Est Marne-la-Vallée, Institut Français d'Urbanisme, 39 p + annexes. 
27. Communauté d'agglomération de Caen La mer (2011) La LNPN: une priorité pour renforcer l'accessibilité et le rayonnement métropolitain de Caen la mer. Ligne Nouvelle Paris Normandie Débat Public, Cahier d'acteur, $4 \mathrm{p}$.

28. Ville de Roanne (2011) Le tracé par Roanne, une évidence pour 3 fonctions ! Projet de Ligne à Grande Vitesse POCL, Cahier d'acteur $\mathrm{n}^{\circ} 1,4 \mathrm{p}$.

29. Perrin J (2009) La Région Ile-de-France et les enjeux liés aux projets de ligne à grande vitesse - Point de vue francilien sur les fonctionnalités de la « grande vitesse ». Mémoire de Master 2, Université de Marne-la-Vallée, IUP - ENPC, 107 p.

30. Perrin J (2012) Géographie de la grande vitesse ferroviaire dans la métropole parisienne - Dessertes et gares d'interconnexion en question. Colloque de 1'AHICF 2012, 22-24 novembre, Paris.

31. Bonnafous A (1980) Rhône-Alpes, capitale Paris ? Les effets prévisibles du TGV. Revue de géographie de Lyon 55(3): 233-240.

32. Bonnafous A (1987) The regional impact of the TGV. Transportation 14: $127-137$

33. Cointet-Pinell O, Plassard F, Michaeli D (1988) Les effets socioéconomiques du TGV en Bourgogne et Rhône-Alpes. DATAR, INRETS, OEST, SNCF, $20 \mathrm{p}$.

34. Bazin S, Beckerich C, Blanquart C, Vandenbossche L (2011) Grande vitesse ferroviaire et développement économique local : une revue de la littérature. Recherche Transports Sécurité 27(106): 215-238.

35. Puga D (2002) European regional policies in light of recent location theories. Journal of economic geography 2(4): 373-406.

36. Troin JF (1997) Les gares TGV et le territoire : débat et enjeux. Annales de Géographie 106(593-594): 34-50.

37. Rietveld P, Bruinsma F, Van Delft HT, Ubbels B (2001) Economic impacts of high speed trains. Experiences in Japan and France: expectations in The Netherlands. Serie Research Memoranda (Faculteit der Economische Wetenschappen en Bedrijfskunde), $20 \mathrm{p}$.

38. Haynes KE (1997) Labor markets and regional transportation improvements: the case of high speed trains. An introduction and review. The annals of regional science 31(1): 57-76.

39. Christaller W (1964) Some considerations of tourism location in Europe: the peripheral regions-underdeveloped countries-recreation areas. Papers in Regional Science12(1): 95-105.

40. Daniels MJ (2007) Central place theory and sport tourism impacts. Annals of Tourism Research 34(2): 332-347.

41. Stock M (2012) Le problème du tourisme dans le modèle des lieux centraux. Réflexions critiques. HALSHS, $21 \mathrm{p}$.
42. Carrouet G (2013) Du TGV Rhin-Rhône au territoire Rhin-Rhône : réticularité, mobilité et territorialité dans un espace intermédiaire. Thèse en géographie et Aménagement, de l'Université de Bourgogne, $518 \mathrm{p}$.

43. SNCF (2011) Révision du Schéma Directeur de la Région Ile-deFrance - Contribution de SNCF, $66 \mathrm{p}$

44. Institut d'Aménagement et d'Urbanisme (IAU) Ile-de-France (2010) Aménagement et développement touristique, Contribution au schéma régional de développement du tourisme et des loisirs en Ile-de-France 2010-2020, $66 \mathrm{p}$.

45. Facchinetti-Mannone V (2005) La nodalité des gares TGV périphériques. Les Cahiers Scientifiques des Transports 48: 45-58.

46. Delaplace M, Perrin J (2012) Quel développement autour du binôme Parc de loisirs et gare TGV ? Les cas de Disneyland $\mathbb{R}$ Paris et du Futuroscope. Colloque « 20 ans de Transformations Economiques et Sociales au Val d'Europe», Val d'Europe, 19-20 décembre.

47. Office du Tourisme et des Congrès de Paris (OTCP) (2011) 40 ans de tourisme à Paris. parisinfo.com, $54 \mathrm{p}$.

48. IAU Ile-de-France (2007) La réorganisation des sièges sociaux et des activités tertiaires centrales des grandes entreprises. Principes, et pratiques, conséquences sur l'évolution spatiale en Ile-de-France. $158 \mathrm{p}$.

49. Comité Régional du Tourisme (CRT) Paris Ile-de-France (2011) Repères de l'activité touristique de la destination Paris Ile-deFrance, $4^{\mathrm{e}}$ édition, $90 \mathrm{p}$.

50. Conseil National du Tourisme (CNT) (2010) Le poids économique et social du tourisme. Ministère de l'économie, de l'industrie et de l'emploi, $137 \mathrm{p}$.

51. Pelisson G (2011) Le tourisme d'affaires dans le Grand Paris, pour une nouvelle ambition. Ministère de l'économie, des finances et de l'industrie, $85 \mathrm{p}$.

52. CRT Paris Ile-de-France (2011) Stratégie Régional de Développement du Tourisme et des Loisirs 2011-2016 (SRDTL), Région Ile-de-France, $42 \mathrm{p}$.

53. Chambre de Commerce et d'Industrie Paris (CCIP) (2011) Sept conditions pour améliorer l'accueil et la mobilité des touristes en Ile-de-France. Rapport Demonchy, $52 \mathrm{p}$.

54. Jolly S (2011) La problématique territoriale dans les politiques locales de développement touristique : le cas de la métropole parisienne. Premières Doctoriales du Tourisme de la Chaire « Culture, Tourisme, développement », EIREST - Université de Paris 1 Panthéon-Sorbonne.

55. Brault F (2010) La bataille du rail low cost n'a pas encore commencé ! Revues Espaces 282: 37-39. 